\title{
Relevant associations of the glucokinase regulatory protein/glucokinase gene variation with TAG concentrations in a high-cardiovascular risk population: modulation by the Mediterranean diet
}

\author{
Mercedes Sotos-Prieto ${ }^{1,2,3}$, Marisa Guillén ${ }^{1}$, Jose Vicente Sorli ${ }^{1,2}$, Olga Portolés ${ }^{1}$, \\ Patricia Guillem-Saiz ${ }^{1,2}$, Jose Ignacio Gonzalez ${ }^{1,2}, \mathrm{Lu}_{\mathrm{Qi}}{ }^{3}$ and Dolores Corella ${ }^{1,2 *}$ \\ ${ }^{1}$ Genetic and Molecular Epidemiology Unit, Department of Preventive Medicine and Public Health, School of Medicine, \\ University of Valencia, Blasco Ibañez, 15. 46010-Valencia, Spain \\ ${ }^{2}$ CIBER Fisiopatología de la Obesidad y Nutrición, Valencia, Spain \\ ${ }^{3}$ Department of Nutrition and Epidemiology, Harvard School of Public Health, Boston, MA, USA
}

(Submitted 13 June 2011 - Final revision received 8 February 2012 - Accepted 8 February 2012 - First published online 13 April 2012)

\section{Abstract}

The SNP rs1260326 (P446L) and rs1799884 (-30G > A) for the glucokinase regulatory protein $(G C K R)$ and glucokinase $(G C K)$ genes, respectively, have been associated with opposing effects on TAG and glucose concentrations. However, their genetic modulation by diet (dietary patterns or foods) remains to be investigated. We studied 945 high-cardiovascular risk subjects aged 67 (SD 6 ) years who participated in the PREvención con DIeta MEDiterránea-Valencia Study. Demographic, clinical, biochemical and genetic data were obtained. Adherence to the Mediterranean diet (MD) and food intake were measured by validated questionnaires. Carriers of the L allele of $G K C R$ had significantly higher TAG concentrations (PP: 1.34 (SD 0.05) mmol/1 $v$. PL + LL: 1.54 (SD 0.03) mmol/1; P=0.014) and LL carriers had lower glucose concentrations (PL + PP: $6.85(\mathrm{SD} 0.08) \mathrm{mmol} / \mathrm{l} v$. LL: $6 \cdot 40(\mathrm{sD} 0 \cdot 16) \mathrm{mmol} / \mathrm{l} ; P=0 \cdot 032)$ after multivariate adjustment. Conversely, homozygous subjects for the variant allele (A) in the GCK gene had significantly lower TAG (GG + GA: 1.48 (SD 0.03) mmol/1 $v$. AA: $1 \cdot 17(\mathrm{sD} 0 \cdot 18) \mathrm{mmol} / \mathrm{l} ; P=0.033)$ and a higher risk of diabetes (OR 3.3, 95\% CI 1.2, 9.2). Combined effects for both SNP increased TAG concentrations by $37 \%(P=0.033)$. Adherence to the MD modulated the effects of $G C K R$ polymorphism on TAG: subjects with genetic risk had lower TAG (L-allele carriers; PP: 1.48 (SD 0.14) mmol/l $v$. PL + LL: 1.51 (sD 0.08) mmol/l; $P=0.917)$ compared with those with a higher adherence. Analysis of the joint effects of the GCKR and individual food items identified significant associations (olive oil ( $P=0.035)$, vegetables $(P=0.012)$, red meat $(P=0.017)$, butter $(P=0.039)$, sweetened carbonated beverages $(P=0.036)$ and nuts $(P=0 \cdot 038)$ ). In conclusion, we found that $\operatorname{rs} 1260326(G C K R)$ is significantly associated with higher TAG concentrations, but is modulated by adherence to the MD.

\section{Key words: Glucokinase regulatory protein: TAG concentration: Nutrigenetics: Mediterranean diet}

The glucokinase $(G C K)$ gene is a regulator of glucose storage and disposal in the liver, and its activity is modulated by binding to the glucokinase regulatory protein (GCKR). Mice deficient in GCKR show reduced expression of GCK and defects in the clearance of glucose, but maintain a normal activity of $\mathrm{GCK}^{(1)}$. Overexpression by the adenoviral-mediated hepatic overproduction of GCKR results in increased GCK activity but lower levels of fasting glucose, while overexpression of GCK in the liver leads to a decrease in glucose concentrations and an increase in TAG concentrations ${ }^{(2,3)}$. Taken together, these data strongly support a role for these genes in determining plasma TAG and glucose concentrations in human subjects.
Initial studies ${ }^{(4,5)}$ have reported an intronic polymorphism of GCKR (rs780094) to be associated with TAG concentrations in populations of European origin. Subsequent research has found similar associations for TAG and opposing effects on glucose concentrations ${ }^{(6-8)}$. Moreover, Orho-Melander et al. ${ }^{(5)}$ suggested rs1260326 (P446L) as the functional SNP for the observed effects of the intronic rs780094.

The common variant $-30 \mathrm{G}>\mathrm{A}(\mathrm{rs} 1799884)$ in the GCK gene promoter region has been associated with an increased risk of diabetes ${ }^{(9)}$, hyperglycaemia ${ }^{(10-12)}$, impaired $\beta$-cell function ${ }^{(10-13)}$ and lower TAG concentrations. An initial study on the combined effects of these polymorphisms has shown the additive effect of GCKR (446L) and GCK (-30G)

Abbreviations: GCK, glucokinase; GCKR, glucokinase regulatory protein; MD, Mediterranean diet.

*Corresponding author: D. Corella, fax +34 963864166, email dolores.corella@uv.es 
alleles in conferring lower fasting glucose and insulin levels, but higher levels of $\mathrm{TAG}^{(9)}$. However, the effects of genetic variants in both genes in determining fasting TAG and glucose concentrations in Mediterranean populations, as well as the possible gene-diet interactions regarding adherence to the Mediterranean diet (MD) and its specific food items, remain to be investigated. We have shown that the MD has a beneficial impact on TAG concentrations and on other biochemical parameters related to a higher risk of $\mathrm{CVD}^{(14)}$. Since diet is one of the most important environmental factors in determining plasma TAG and glucose concentrations, we hypothesise that adherence to the MD may be directly involved in the genetic modulation of different intermediate phenotypes of the GCK and GCKR genes. Although two recent studies have analysed gene-diet interactions for these genes $^{(15,16)}$, neither of them has focused on examining the effects of the MD in such interactions.

The aims of the present study are as follows: first, to analyse the effects of GCKR P446L (rs1260326) and GCK - 30G > A (rs1799884) on fasting TAG and glucose concentrations in a high-cardiovascular risk Mediterranean population; second, to study the potential modulation of the effect of GCKR SNP on TAG concentrations by adherence to the MD; third, to analyse the joint association and the potential interaction of each food item of the MD in relation to TAG concentrations.

\section{Subjects and methods}

We studied 945 unrelated consecutive subjects (340 men and 605 women), aged 55-80 years, who participated in the PREvención con DIeta MEDiterránea Study. These subjects had been recruited in the Valencia Region (Spain) from October 2003 to September 2008, and details of this study have been reported elsewhere ${ }^{(14)}$. Briefly, high-risk participants were selected by physicians in primary care centres. Eligible subjects were elderly community-dwelling persons who fulfilled at least one of two criteria: type 2 diabetes or three or more CVD risk factors (current smoking, hypertension, dyslipidaemia, overweight or a family history of premature CVD). Exclusion criteria were based on a personal history of CVD, any severe chronic illness, and drug or alcohol addiction. Here, we included data from the baseline cross-sectional examination. The present study was conducted according to the guidelines laid down in the Declaration of Helsinki and all procedures involving human subjects were approved by the Institutional Review Board of the Valencia University. Written informed consent was obtained from all subjects.

\section{Anthropometric, biochemical, clinical and lifestyle determinations}

Anthropometric variables, such as height, weight and waist circumference, were measured by standard techniques ${ }^{(14)}$ BMI was calculated as weight $(\mathrm{kg})$ divided by height $\left(\mathrm{m}^{2}\right)$. Fasting blood samples were obtained for each participant, and stored at $-80^{\circ} \mathrm{C}$ until biochemical analyses. Fasting glucose, total cholesterol, TAG, HDL-cholesterol and LDL-cholesterol were determined as reported previously ${ }^{(14)}$.
Baseline examination included assessment of standard cardiovascular risk factors, medication use, sociodemographic factors and lifestyle variables, as detailed previously ${ }^{(14)}$. Physical activity was estimated by the Minnesota Leisure-Time Physical Activity Questionnaire ${ }^{(17)}$.

\section{Dietary intake}

We determined food consumption by a previously validated semi-quantitative $\mathrm{FFQ}^{(18)}$. Energy and nutrient intake was calculated from Spanish food composition tables ${ }^{(19)}$. The baseline examination also included the administration of a validated fourteen-item questionnaire that indicated the degree of adherence to the traditional $\mathrm{MD}^{(20)}$. Values of 0 or 1 were assigned to each of the fourteen dietary components. For each of the following categories, one point was given: (1) the use of olive oil as the principal source of fat for cooking; (2) $\geq 4$ tablespoons (one tablespoon $=13.5 \mathrm{~g}$ ) of olive oil/d (including that used in frying, salads, meals eaten away from home, etc.); (3) $\geq 2$ servings of vegetables/d; (4) $\geq 3$ pieces of fruit/d; $(5)<1$ serving of red meat or sausages/d; (6) $<1$ serving of butter, margarine or cream/d; (7) $<1$ cup (one cup $=100 \mathrm{ml}$ ) of sugar-sweetened beverages/d; (8) $\geq 7$ servings of red wine/week; (9) $\geq 3$ servings of pulses/week; (10) $\geq 3$ servings of fish/week; (11) $<2$ commercial pastries/ week; (12) $\geq 3$ servings of nuts/week; (13) preferring white meat over red meat; or for consuming (14) $\geq 2$ servings/ week of a dish with a traditional sauce of tomatoes, garlic, onion or leeks sautéed in olive oil. If the condition was not met, 0 points were recorded for that category. The greater the score obtained from the questionnaire, the greater the adherence to the MD. Further, we created four groups of adherence to the MD according to the points obtained in this questionnaire: group 1 (3-6 points, $15 \cdot 6 \%$ ), group 2 (7-8 points, $35 \cdot 2 \%$ ), group $3(9-10$ points, $32 \cdot 6 \%)$ and group 4 (11-14 points, $16.6 \%)$, such that group 1 had the lowest adherence to the MD and group 4 had the highest adherence.

\section{SNP analyses}

DNA was extracted from the buffy coat fraction of centrifuged blood using the MagNa Pure LC DNA Isolation Kit (Roche Diagnostics). GCKR P446L (rs1260326) and GCK $-30 \mathrm{~A}>\mathrm{G}$ (rs1799884) were genotyped using Taqman SNP allelic discrimination by means of an ABI 7900 HT (Applied Biosystems). Both genes were in Hardy-Weinberg equilibrium in the study samples $(P=0 \cdot 38$ and $P=0 \cdot 144$, respectively). A concordance rate of $100 \%$ was seen in 200 blinded qualitycontrol samples for all SNP assayed.

\section{Statistical analysis}

Categorical variables were compared using $\chi^{2}$ tests. Student's $t$ test and ANOVA were applied to compare crude means. Dominant, co-dominant and recessive models of inheritance were first tested to determine the effects of the variant allele. TAG concentrations were log-transformed for statistical testing. 
A dominant model for the $\mathrm{P} 446 \mathrm{~L}$ SNP and a recessive model for the $-30 \mathrm{G}>\mathrm{A}$ SNP were the most significant, and these categories (PP $v$. PL + LL and GG $+\mathrm{GA} v$. AA, respectively) were considered for further analyses. We further adjusted for potential confounders using ANCOVA. Joint analysis was performed considering the genotype (PP or PL $+\mathrm{LL}$ ) and each category of the food item using ANCOVA.

In multivariate analysis, the models were adjusted for covariates including age (continuous), BMI ( $\mathrm{kg} / \mathrm{m}^{2}$; continuous), sex, diabetes (yes or no), smoking (current smokers or no smokers) and lipid medication (yes or no). Further adjustment of the models for physical activity and total energy intake did not change the statistical significance of the results. Logistic regression methods were also used to estimate the OR of hypertriacylglycerolaemia and diabetes associated with the polymorphisms. Univariate and multivariate logistic regression models including the potential confounders were fitted. Statistical analyses were performed using the SPSS package, version 15.0 (SPSS). Statistical significance was set at the 0.05 level, and all tests were two-tailed.

\section{Results \\ Characteristics of the population}

Clinical, biochemical, dietetic and genetic characteristics are shown in Table 1. Participants were high-cardiovascular risk subjects and then, prevalence of hypertriacylglycerolaemia and diabetes was high. The mean of the adherence to the MD was 8.5 points. The percentages of positive points for the most representative food items of the MD are listed in Table 1.

\section{Association between the glucokinase regulatory protein}

gene (rs1260326) (P446L) and glucokinase gene

(rs1799884) (-30G > A) polymorphisms with fasting TAG and glucose concentrations

The GCKR (P446L) SNP showed a significant association with TAG concentrations (Table 2). Subjects who were heterozygous and homozygous for the minor allele (L) showed higher TAG concentrations ( $P$ crude model $=0 \cdot 006$ ). The best model identified for this genetic variant was dominant $(P=0 \cdot 002)$. Multivariate adjustment for age, sex, BMI, smoking, diabetes and lipid medication did not significantly change the results $(P=0 \cdot 014)$. This association was homogeneous in males and females. No associations were found for other lipids parameters and the GCKR polymorphism (data not shown).

No significant association was found between the $-30 G>A$ ANP in the GCK gene and TAG concentrations in the crude analysis. However, after multivariate adjustment, homozygous subjects for the minor allele (AA) presented statistically lower TAG concentrations than the GG + GA subjects $(P=0.033)$. No statistical association was found between either of the SNP analysed and glucose concentrations.

We also examined the combined effects for each genotype and found an additive effect on TAG concentrations. After full multivariate adjustment, we found that subjects with the highest genetic risk for TAG, namely PL/LL $(G C K R)+\mathrm{GG} /$ GA $(G C K)$, had a $37 \%$ higher TAG concentration compared with those with a genetic-free risk (i.e. PP $(G C K R)+\mathrm{AA}$ $(G C K))(P=0 \cdot 033)$.

No combined effects were found for glucose concentrations. We also studied the gene $\times$ gene interaction, but no statistically significant interactive effects were found.

We also estimated the association with the risk of hypertriacylglycerolaemia and diabetes. We found a significantly higher risk for hypertriacylglycerolaemia in the PL + LL subjects (OR 1.86, 95\% CI 1.33, 2.61) than in the PP subjects.

Table 1. Anthropometric, clinical, biochemical, lifestyle and genetic characteristics of the high-cardiovascular risk Mediterranean population

(Mean values, standard deviations, number of subjects and percentages)

\begin{tabular}{|c|c|c|}
\hline Variables & Mean & SD \\
\hline \multicolumn{3}{|l|}{ Continuous variables } \\
\hline \multicolumn{3}{|l|}{$\operatorname{Sex}(n)$} \\
\hline Male & \multicolumn{2}{|c|}{340} \\
\hline Female & \multicolumn{2}{|c|}{605} \\
\hline Age (years) & $67 \cdot 3$ & $6 \cdot 2$ \\
\hline Weight (kg) & $77 \cdot 4$ & $12 \cdot 4$ \\
\hline $\mathrm{BMI}\left(\mathrm{kg} / \mathrm{m}^{2}\right)$ & $30 \cdot 9$ & $5 \cdot 1$ \\
\hline Waist $(\mathrm{cm})$ & $104 \cdot 3$ & $11 \cdot 8$ \\
\hline $\mathrm{TC}(\mathrm{mmol} / \mathrm{l})$ & $5 \cdot 38$ & 1.03 \\
\hline HDL-C (mmol/l) & 1.37 & 0.36 \\
\hline LDL-C (mmol/l) & 3.34 & 0.93 \\
\hline TAG (mmol/l) & 1.48 & 0.91 \\
\hline Fasting glucose (mmol/l) & 6.83 & $2 \cdot 22$ \\
\hline $\mathrm{SBP}(\mathrm{mmHg})$ & 147 & 22 \\
\hline $\mathrm{DBP}(\mathrm{mmHg})$ & 82 & 11 \\
\hline Score for MD adherence* & 8.5 & $2 \cdot 0$ \\
\hline \multicolumn{3}{|l|}{ Energy intake } \\
\hline $\mathrm{kJ}$ & 9228 & 2454 \\
\hline \multirow[t]{2}{*}{ kcal } & 2204 & 586 \\
\hline \multirow{2}{*}{\multicolumn{3}{|c|}{ Categorical variables }} \\
\hline & & \\
\hline Use of olive oil as principal source of fat & 754 & $86 \cdot 3$ \\
\hline Olive oil $\geq 4 \mathrm{tbs} / \mathrm{d}$ & 708 & $81 \cdot 2$ \\
\hline Vegetables $\geq 2$ servings/d & 527 & $46 \cdot \overline{7}$ \\
\hline Fruit consumption $\geq 3$ servings $/ d$ & 400 & $45 \cdot 9$ \\
\hline Red meat $<1$ serving/d & 418 & 47.9 \\
\hline Butter $<1$ serving $/ \mathrm{d}$ & 793 & $90 \cdot 7$ \\
\hline Sugar-sweetened beverages $<1$ serving $/ d$ & 758 & $86 \cdot 8$ \\
\hline Wine $\geq 7$ cups/week & 179 & $20 \cdot 6$ \\
\hline Legumes $\geq 3$ servings/week & 220 & $25 \cdot 2$ \\
\hline Fish $\geq 3$ servings/week & 703 & $80 \cdot 4$ \\
\hline Commercial pastries $<2$ servings/week & 602 & 68.9 \\
\hline Nuts $\geq 3$ servings/week & 229 & $26 \cdot 3$ \\
\hline Preferring white meat over red meat & 642 & 73.5 \\
\hline Traditional sauce 'sofrito' $\geq 2$ servings/week & 482 & $55 \cdot 4$ \\
\hline Current smokers & 109 & 11.9 \\
\hline Diabetes & 448 & 48.5 \\
\hline Hypertriacylglycerolaemia & 266 & $28 \cdot 6$ \\
\hline \multicolumn{3}{|l|}{ GCKR (rs1260326) (P446L) polymorphism } \\
\hline PP & 284 & $29 \cdot 6$ \\
\hline PL & 488 & $50 \cdot 9$ \\
\hline LL & 187 & 19.5 \\
\hline \multicolumn{3}{|l|}{ GCK (rs1799884) $(-30 \mathrm{G}>\mathrm{A})$ polymorphism } \\
\hline$G G$ & 598 & $64 \cdot 6$ \\
\hline GA & 300 & $32 \cdot 4$ \\
\hline AA & 27 & $2 \cdot 9$ \\
\hline
\end{tabular}

TC, total cholesterol; HDL-C, HDL-cholesterol; LDL-C, LDL-cholesterol; SBP, systolic blood pressure; DBP, diastolic blood pressure; MD, Mediterranean diet; tbs, tablespoon; GCKR, glucokinase regulatory protein; GCK, glucokinase.

* Score for MD adherence: 14-unit MD score (ranging from 0 (minimum) to 14 points (maximum)). 
Table 2. Association between fasting TAG and glucose concentrations (mmol/l) and $P 446 \mathrm{~L}$ (rs1260326) glucokinase regulatory protein $(G C K R)$ gene, rs $1799884(-30 G>A)$ glucokinase $(G C K)$ gene and combined effect (GCKR+GCK) (Mean values and standard deviations or standard errors)

\begin{tabular}{|c|c|c|c|c|c|c|c|c|c|}
\hline & \multicolumn{9}{|c|}{ P446L (rs1260326) GCKR gene } \\
\hline & \multicolumn{2}{|c|}{$\mathrm{PP}(n 288)$} & \multicolumn{2}{|c|}{$\mathrm{PL}(n$ 488) } & \multicolumn{2}{|c|}{$\mathrm{LL}(n 187)$} & \multirow[b]{2}{*}{$P^{\star}$} & \multirow[b]{2}{*}{$P_{\text {dominant }}$} & \\
\hline & Mean & SE & Mean & SE & Mean & SE & & & \\
\hline \multicolumn{10}{|l|}{ TAG (mmol/l) } \\
\hline Crude & & & \multirow{2}{*}{\multicolumn{2}{|c|}{1.51}} & \multirow{2}{*}{\multicolumn{2}{|c|}{1.57}} & 0.006 & 0.002 & \\
\hline Mean & \multicolumn{2}{|c|}{1.33} & & & & & & & \\
\hline SD & \multicolumn{2}{|c|}{0.58} & \multicolumn{2}{|c|}{0.88} & \multicolumn{2}{|c|}{0.90} & & & \\
\hline Model 1† & 1.33 & 0.05 & 1.50 & 0.04 & 1.57 & 0.06 & 0.012 & 0.004 & \\
\hline Model $2 \ddagger$ & 1.34 & 0.05 & 1.50 & 0.04 & 1.58 & 0.07 & 0.035 & 0.014 & \\
\hline \multicolumn{10}{|c|}{ Glucose $(\mathrm{mmol} / \mathrm{l})$} \\
\hline Crude & & & & & & & 0.151 & 0.068 & \\
\hline Mean & \multirow{2}{*}{\multicolumn{2}{|c|}{$\begin{array}{l}6.78 \\
2.08\end{array}$}} & \multirow{2}{*}{\multicolumn{2}{|c|}{$\begin{array}{l}6.89 \\
2.25\end{array}$}} & \multirow{2}{*}{\multicolumn{2}{|c|}{$\begin{array}{l}6.53 \\
1.90\end{array}$}} & & & \\
\hline SD & & & & & & & & & \\
\hline Model $1 \dagger$ & $6 \cdot 77$ & 0.13 & 6.91 & 0.10 & 6.46 & $0 \cdot 17$ & 0.071 & 0.866 & \\
\hline \multirow[t]{4}{*}{ Model $2 \ddagger$} & $6 \cdot 71$ & 0.12 & 6.84 & 0.09 & 6.55 & 0.14 & 0.224 & 0.141 & \\
\hline & \multicolumn{8}{|c|}{ rs1799884 $(-30 G>A) G C K$ gene } & \\
\hline & \multicolumn{2}{|c|}{$\mathrm{GG}(n 598)$} & \multicolumn{2}{|c|}{$\mathrm{GA}(n 300)$} & \multicolumn{2}{|c|}{$\mathrm{AA}(n 27)$} & & & \\
\hline & Mean & SE & Mean & SE & Mean & SE & $P^{\star}$ & $P_{\text {recessive }}$ & \\
\hline TAG $(\mathrm{mmol} / \mathrm{l})$ & & & & & & & & & \\
\hline Crude & & & & & & & 0.258 & 0.1 & \\
\hline Mean & & & & & & & & & \\
\hline $\mathrm{SD}$ & & & & & & & & & \\
\hline Model 1† & 1.47 & 0.03 & 1.48 & 0.05 & $1 \cdot 18$ & 0.16 & 0.199 & 0.027 & \\
\hline Model $2 \ddagger$ & 1.48 & 0.04 & 1.46 & 0.05 & $1 \cdot 17$ & 0.18 & 0.095 & 0.033 & \\
\hline Glucose $(\mathrm{mm}$ & & & & & & & & & \\
\hline Crude & & & & & & & 0.517 & 0.323 & \\
\hline Mean & & & & & & & & & \\
\hline SD & & & & & & & & & \\
\hline Model $1 \dagger$ & 6.74 & 0.09 & $6 \cdot 80$ & 0.13 & $7 \cdot 18$ & 0.43 & 0.608 & 0.347 & \\
\hline Model $2 \ddagger$ & 6.75 & 0.08 & 6.78 & 0.12 & 6.09 & 0.40 & 0.262 & $0 \cdot 105$ & \\
\hline & & & & ined ef & $\mathrm{t}(G C K)$ & ominar & $-G C K \mathrm{r}$ & ssive) & \\
\hline & & & $\begin{array}{r}\mathrm{PP}+ \\
\mathrm{AA}\end{array}$ & $\begin{array}{l}A+ \\
253)\end{array}$ & $\begin{array}{c}(\mathrm{PL}+ \\
\mathrm{AA}\end{array}$ & $\begin{array}{l}\text { L) }+ \\
608)\end{array}$ & $\begin{aligned} & (\mathrm{PP} \\
\text { (GG } & \end{aligned}$ & $\begin{array}{l}\text { LL) }+ \\
\text { A) }(n 14)\end{array}$ & \\
\hline & Mean & $\overline{S E}$ & Mean & $\overline{\mathrm{SE}}$ & Mean & SE & Mean & SE & $P$ for joint effect \\
\hline TAG $(\mathrm{mmol} / \mathrm{l})$ & & & & & & & & & \\
\hline Crude & & & & & & & & & 0.012 \\
\hline Mean & & & & & & & & & \\
\hline SD & & & & & & & & & \\
\hline Model 1† & 1.06 & 0.24 & 1.35 & 0.05 & 1.27 & 0.22 & 1.53 & 0.03 & 0.010 \\
\hline Model $2 \ddagger$ & $1 \cdot 11$ & 0.27 & 1.36 & 0.06 & $1 \cdot 21$ & 0.24 & 1.52 & 0.04 & 0.033 \\
\hline Glucose $(\mathrm{mm}$ & & & & & & & & & \\
\hline Crude & & & & & & & & & 0.799 \\
\hline Mean & & & & & & & & & \\
\hline SD & & & & & & & & & \\
\hline Model 1† & 7.32 & 0.64 & 6.73 & 0.14 & 7.05 & 0.59 & $6 \cdot 78$ & 0.09 & 0.784 \\
\hline Model $2 \ddagger$ & 6.49 & 0.58 & 6.73 & 0.12 & 5.74 & 0.56 & $6 \cdot 77$ & 0.08 & 0.311 \\
\hline
\end{tabular}

* $P$ value for comparisons between genotypes and TAG concentrations. $P$ value for log TAG.

$\dagger$ Adjusted for age, sex and BMI.

† Model 1 + smoking + diabetes + lipid medication.

Further multivariate adjustment did not change the statistical significance $(P=0 \cdot 001)$. The $G C K$ SNP was not associated with hypertriacylglycerolaemia risk. The regression logistic model for combined effects had a low number of participants in both categories of reference for comparisons; therefore, we could not find statistically significant results (see Table S1 of the supplementary material, available online at http://www. journals.cambridge.org/bjn). Opposing effects were observed for type 2 diabetes mellitus risk in relation to the L446P and $-30 \mathrm{G}>\mathrm{A}$ polymorphisms. The LL subjects $(G C K R)$ had a lower estimated risk for type 2 diabetes mellitus, while the AA subjects had a statistically higher risk (LL $(G C K R)$ : OR $_{\text {adjusted }} 0.80,95 \%$ CI 0.66, 0.96; $P=0.017$ and AA $(G C K)$ : OR $_{\text {adjusted }} 3 \cdot 3,95 \%$ CI $1 \cdot 2,9 \cdot 2 ; P=0.023$ ) (see Table S2 of 
the supplementary material, available online at http://www. journals.cambridge.org/bjn).

Interaction between glucokinase regulatory protein gene (rs1260326) (P446L) polymorphism and adherence to the Mediterranean diet in determining TAG concentrations

We tested whether adherence to the MD modified the associations between the P446L SNP and TAG concentrations, according to groups of adherence. Based on the score obtained in the questionnaire of adherence to the MD, four categories of adherence to the MD were considered. We found statistically significant differences in TAG concentrations by genotype among those with a lower adherence to the MD (group 1-group 2-group 3; $P=0 \cdot 011$ ). In contrast, no differences were found between genotypes and TAG concentrations in the group of higher adherence to the MD $(P=0.917$; Fig. 1). However, the test for interaction was not significant.

\section{Joint associations and interactions between glucokinase} regulatory protein gene (rs1260326) (P446L)

polymorphism and food items of the Mediterranean diet in determining TAG concentrations

We examined the joint associations between the P446L SNP and the major foods that characterise the MD in relation to TAG concentrations (Table 3). Statistically significant joint associations were found for olive oil, vegetables, meat, butter, sweetened carbonated beverages and nuts $(P=0.035$, $P=0.012, P=0.017, P=0.039, P=0.036$ and $P=0.038$, respectively). Subjects with the highest risk (genetic risk $(P L+L L)$ ) and who had a high consumption of sweetened carbonated beverages $(>1$ per d) had $0.19 \mathrm{mmol} / \mathrm{l}$ higher TAG

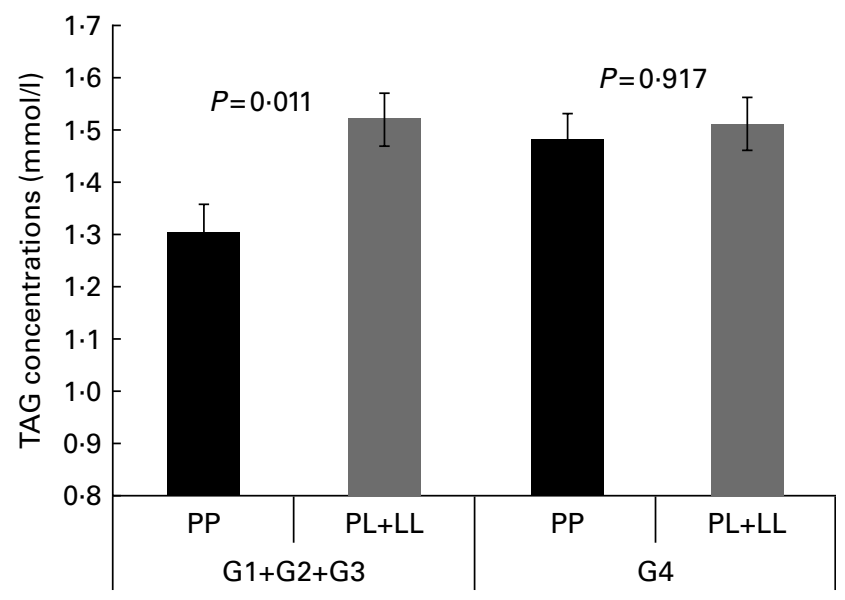

Adherence to the Mediterranean diet

Fig. 1. Gene $\times$ diet interaction for TAG concentrations according to adherence to the Mediterranean diet (AMD). Adjusted means of TAG are shown by the GCKR polymorphism according to the strata of AMD. Means were adjusted by sex, age, smoking, BMI, diabetes and lipid medication. $P$ value for interaction terms between TAG concentrations and GCKR polymorphism (as a dominant pattern) was obtained in the hierarchical multivariate interaction model ( $P$ for GCKR (P446L) $\times$ AMD interaction $=0.346)$. $P$ value for $\log$ TAG. G1+G2 + G3, groups 1, 2 and 3 of AMD; G4, group 4 of AMD.

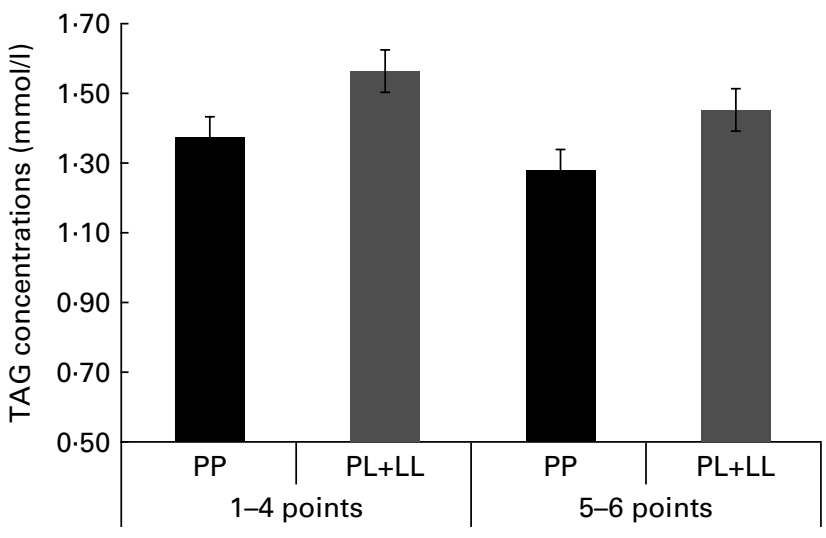

Mini-score of adherence to the Mediterranean diet

Fig. 2. TAG concentrations according to the joint effects of adherence to the six-point score and the genetic dominant pattern (PP, $\mathrm{PL}+\mathrm{LL}$ ). The analyses were adjusted for sex, age, BMI, smoking, diabetes and lipid medication $(P$ for joint effects $=0.027)$.

concentrations than those with the lowest allele of risk (PP) and lowest consumption of sweetened carbonated beverages ( $\leq 1$ per $\mathrm{d}$ ). Similar results were observed for butter, margarine or cream consumption per $\mathrm{d}$, although with lower effects on the reduction of TAG concentrations (2.5\%).

For the other main items that characterise the MD, subjects with the highest genetic risk and lower consumption of olive oil had $8.2 \%(0.25 \mathrm{mmol} / \mathrm{l})$ higher TAG concentrations than did those with no allele risk and higher consumption of olive oil ( $\geq 4$ tablespoons) ( $P=0.033$ ). Similarly, those who consumed less than two servings per $d$ of vegetables, or more than one serving per $d$ of red meat, or less than three servings of nuts per week had $0.30(23 \%), 0.06(4.2 \%)$ or $0 \cdot 11(8 \cdot 2 \%) \mathrm{mmol} / 1$ higher TAG concentrations, respectively. All of the means were multivariate adjusted. Further adjustment for energy intake did not change the statistical significance of the results.

We further assessed the potential interactions between the P446L SNP and the major foods characterising the MD in relation to TAG concentrations. No significant interactions were found. We also analysed the specific effect of each food item on TAG concentrations, independently of the genotype. Significant associations were found (inverse) for vegetables and meat consumption $(P=0.022$ and $P=0.035$, respectively), and these effects were enhanced when genotype was considered ( $P=0 \cdot 012$ and $P=0 \cdot 017$, respectively).

We tried to identify the main items of the MD that specifically could be associated with the genetic risk and TAG concentrations. We created a mini-score with the six items that were significant in the first analysis (olive oil, vegetables, red meat, butter, sweetened carbonated beverages and nuts). In a further analysis, we found a significant joint association. The subjects with the highest genetic risk $(\mathrm{PL}+\mathrm{LL})$ and lower food item consumption (1-4 points) had $22.2 \%$ higher TAG concentrations. In a stratified analysis, we did not find any differences in TAG concentrations between genotypes in those subjects who consumed five or six items of this mini-score, while significant differences were observed when the consumption was one to four items (Fig. 2). 
Table 3. Interaction and joint effects between components of the score of adherence to the Mediterranean diet and the glucokinase regulatory protein gene polymorphism on TAG concentrations ( $\mathrm{mmol} / \mathrm{l})$

(Mean values with their standard errors)

\begin{tabular}{|c|c|c|c|c|c|c|}
\hline & \multicolumn{2}{|c|}{ PP } & \multicolumn{2}{|c|}{$\mathrm{PL}+\mathrm{LL}$} & \multirow[b]{2}{*}{$P$ for joint effects ${ }^{*}$} & \multirow[b]{2}{*}{$P$ food item } \\
\hline & Mean & SE & Mean & SE & & \\
\hline \multicolumn{7}{|c|}{ Olive oil (tbs/d) } \\
\hline$<4$ & 1.44 & 0.08 & 1.63 & 0.07 & 0.035 & 0.116 \\
\hline$\geq 4$ & 1.30 & 0.06 & 1.49 & 0.04 & & \\
\hline \multicolumn{7}{|c|}{ Vegetables (servings/d) $\dagger$} \\
\hline$<2$ & 1.40 & 0.07 & 1.59 & 0.05 & 0.012 & 0.022 \\
\hline$\geq 2$ & 1.29 & 0.06 & 1.47 & 0.04 & & \\
\hline \multicolumn{7}{|c|}{ Fruit (including natural fruit juice, servings/d) $\ddagger$} \\
\hline$<3$ & $1 \cdot 33$ & 0.06 & 1.51 & 0.05 & 0.084 & 0.931 \\
\hline$\geq 3$ & 1.34 & 0.06 & 1.52 & 0.05 & & \\
\hline \multicolumn{7}{|c|}{$\begin{array}{l}\text { Red meat (hamburgers or meat products, } \\
\text { servings/d) }\end{array}$} \\
\hline$\geq 1$ & 1.24 & 0.09 & 1.41 & 0.08 & 0.017 & 0.035 \\
\hline$<1$ & 1.36 & 0.06 & 1.54 & 0.04 & & \\
\hline \multicolumn{7}{|c|}{ Butter, margarine or cream (servings/d)\| } \\
\hline$\geq 1$ & $1 \cdot 20$ & 0.11 & 1.38 & 0.10 & 0.039 & 0.145 \\
\hline$<1$ & 1.35 & 0.06 & 1.53 & 0.04 & & \\
\hline \multicolumn{7}{|c|}{ Sweetened carbonated beverages (servings/d) } \\
\hline$\geq 1$ & $1 \cdot 28$ & 0.10 & 1.47 & 0.09 & 0.036 & 0.896 \\
\hline$<1$ & 1.34 & 0.06 & 1.53 & 0.04 & & \\
\hline \multicolumn{7}{|c|}{ Wine (servings/week) ${ }^{\star \star}$} \\
\hline$<7$ & 1.32 & 0.06 & 1.42 & 0.01 & 0.051 & 0.230 \\
\hline$\geq 7$ & 1.42 & 0.09 & 1.60 & 0.08 & & \\
\hline \multicolumn{7}{|c|}{ Legumes (servings/week)†† } \\
\hline$<3$ & 1.33 & 0.06 & 1.52 & 0.04 & 0.116 & 0.703 \\
\hline$\geq 3$ & 1.34 & 0.06 & 1.53 & 0.06 & & \\
\hline \multicolumn{7}{|c|}{ 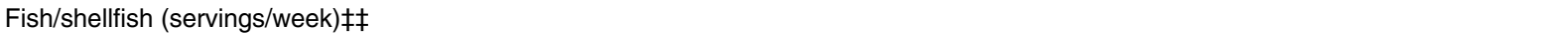 } \\
\hline$<3$ & 1.32 & 0.06 & 1.50 & 0.05 & 0.104 & 0.541 \\
\hline$\geq 3$ & 1.35 & 0.06 & 1.53 & 0.05 & & \\
\hline \multicolumn{7}{|c|}{ Sweets or pastries (servings/week) §§ } \\
\hline$<2$ & 1.34 & 0.07 & 1.52 & 0.06 & $0 \cdot 101$ & 0.476 \\
\hline$\geq 2$ & 1.33 & 0.06 & 1.52 & 0.04 & & \\
\hline \multicolumn{7}{|c|}{ Nuts (servings/week) ${ }^{\| \prime \prime}$} \\
\hline$<3$ & $1 \cdot 31$ & 0.06 & 1.50 & 0.04 & 0.038 & 0.314 \\
\hline$\geq 3$ & 1.38 & 0.07 & 1.57 & 0.06 & & \\
\hline \multicolumn{7}{|c|}{$\begin{array}{l}\text { Consumption preferentially of chicken, rabbit or } \\
\text { turkey instead of veal, pork, hamburger, } \\
\text { sausage } \uparrow\end{array}$} \\
\hline No & $1 \cdot 27$ & 0.08 & 1.45 & 0.06 & 0.099 & 0.480 \\
\hline Yes & 1.35 & 0.06 & 1.54 & 0.04 & & \\
\hline \multicolumn{7}{|c|}{ Vegetables, pasta, rice with sofritos (servings/week) ${ }^{\star \star \star}$} \\
\hline$<2$ & 1.32 & 0.06 & 1.51 & 0.05 & 0.088 & 0.693 \\
\hline$\geq 2$ & 1.34 & 0.06 & 1.52 & 0.04 & & \\
\hline
\end{tabular}

tbs, Tablespoon.

${ }^{*} P$ value for log TAG. The analyses were adjusted for sex, age, BMI, smoking, lipid medication and diabetes.

† Serving of vegetables is $200 \mathrm{~g}$

¥Serving of fruit is $100-250 \mathrm{~g}$.

$\S$ Serving of red meat is $100-150 \mathrm{~g}$.

II Serving of butter, margarine or cream is $12 \mathrm{~g}$

If Serving of sweetened carbonated beverages is $200 \mathrm{ml}$.

${ }^{* *}$ Serving of wine is one cup, $100 \mathrm{ml}$.

t† Serving of legumes is $150 \mathrm{~g}$.

扭 Serving of fish is $100-150 \mathrm{~g}$ and $200 \mathrm{~g}$ (4-5 units) for shellfish

$\S \S$ Serving of sweets or pastries is $50 \mathrm{~g}$ unless other thing is specified in the questionnaire ${ }^{(18)}$.

IIII Serving of nuts is $30 \mathrm{~g}$.

१ १ Serving of poultry is $100-150 \mathrm{~g}$.

${ }^{\star \star \star}$ Serving of sofritos is $200 \mathrm{~g}$

\section{Discussion}

The present study was carried out in a high-cardiovascular risk Mediterranean population. We replicated previously reported associations between the minor allele ( $\mathrm{P}$ variant) of rs1260326 in the GCKR gene and higher TAG concentrations $^{(4-7,9,21-23)}$, and reported for the first time a modulation of this association by adherence to the MD.
The mechanism that may explain the observed results is not yet understood. A recent molecular study ${ }^{(24)}$ proposed a mutational mechanism that could explain the reported association of this variant with raised TAG and lower glucose concentrations. This group found that regulation by physiological concentrations of the phosphate ester fructose-6-phosphate is reduced in the GCKR T-allele Pro446Leu, which results 
indirectly in increased GCK activity ${ }^{(24)}$. Altered GCK regulation in the liver is predicted to enhance glycolytic flux, promoting hepatic glucose metabolism and elevating concentrations of malonyl-CoA, a substrate for de novo lipogenesis. This provides a mutational mechanism for the reported association of this variant with raised TAG and lower glucose levels ${ }^{(24)}$. Taken together, these data support the central position of GCKR in pathways that regulate hepatic TAG as well as glucose metabolism in humans.

With respect to the polymorphism in the GCK gene, most previous studies have analysed its effect on glucose concentrations and have reported significant associations ${ }^{(10,21,25,26)}$. In our population, we found no association with fasting glucose, although the estimated risk of diabetes was lower in the AA subjects, as reported in other studies ${ }^{(10,23)}$.

Opposing effects of GCK and GCKR polymorphisms on TAG concentrations have been reported previously. The minor allele of the $-30 \mathrm{G}>\mathrm{A}$ SNP has been associated with lower TAG concentrations. In the present study, we also found a significant association between the A allele and lower TAG concentrations. Furthermore, when we analysed the combined effect of the GCK gene (recessive model) and GCKR gene (dominant model), we found a potentially increased effect on TAG concentrations in subjects with the AA $(G C K)$ and PP $(G C K R)$ alleles having lower TAG concentrations when compared with those having the PL + LL and GG + GA alleles.

Several studies have reported additive effects of rs7800094 and rs1799884 on fasting glucose concentrations ${ }^{(5,7,9)}$, but this combined effect on TAG concentrations has not been widely studied. Tam et $a{ }^{(21)}$ used additive genetic models in two cohorts of Chinese populations but did not observe an interaction with fasting TAG. Differences between genetic effect size, risk allele frequency, population sample and environmental exposure between European and Asian populations may explain this discrepancy. Additional interaction analyses in further studies are warranted to validate the present findings.

Regarding the gene-diet interaction, we investigated the potential joint effects of genetic variants and the MD on TAG concentrations. We reported a significant difference in TAG between PP $v$. PL + LL in subjects with a lower adherence to the MD, while this effect was not found in the group with a high adherence to the MD. Supporting the presence of gene-diet interactions involving the GCKR gene, two recent studies $^{(15,16)}$ have reported statistically significant interactions between some SNP in the GCKR gene and dietary intake in determining glucose-related traits. In one of them ${ }^{(15)}$, carried out in fourteen cohorts comprising about 48000 participants of European descent aimed at studying interactions of whole grain intake with loci previously associated in genome-wide association studies with fasting glucose (sixteen loci) and/or insulin (two loci), the strongest interaction with whole grain intake was between rs780094 in the GCKR gene in determining fasting insulin. In the other work ${ }^{(16)}$ carried out in subjects with the metabolic syndrome participating in the LIPGENE study, the authors found significant interactions between the GCKR rs1260326 polymorphism and plasma $n$-3 PUFA levels modulating insulin resistance and inflammatory markers These studies ${ }^{(15,16)}$ did not investigate the potential mechanisms behind the reported gene-diet interactions. In the present study, the first to investigate the combined effects of the MD and the genetic risk of P446L on TAG concentrations, we did not study the biological mechanism to explain the observed results. However, taking into account some previous reports ${ }^{(27,28)}$ regarding the effect of dietary fat on the postprandial lipid response, we may suggest some hypotheses. Perez-Martinez et $a$. $^{(27)}$ studied the postprandial TAG response to a high-fat diet and reported that subjects with the risk allele had higher fasting TAG, hypertriacylglycerolaemia and a greater postprandial TAG response. Another dietary intervention study (postprandial lipid response after a high-fat challenge) ${ }^{(28)}$ also reported results consistent with those published by Perez-Martinez et $a l^{(27)}$. These findings could support the present results with respect to modulation of the genetic susceptibility of TAG concentrations by the profile of the high fat content of the traditional MD (a high percentage of fat intake $(30-35 \%)$ is mostly from MUFA, given the consumption of olive oil ${ }^{(14,20)}$. A high fat content from SFA could have a negative effect on $\mathrm{L}$ carriers with respect to TAG concentrations, while fats from olive oil and nuts (characteristic foods of the MD) could modulate this risk (as shown in our sample for these two individual items). In the present study, we showed a significant joint association (gene-food items of the MD) between TAG concentration and the consumption of olive oil, vegetables and nuts, as well as the low consumption of meat products, butter and sweetened beverages. The MD is well known to have beneficial effects on lipid metabolism as well as on other parameters ${ }^{(29,30)}$. Currently, the importance of nutrigenomics and personalised diets based on genetics is gaining attention ${ }^{(31)}$. Therefore, a number of studies have analysed the potential modulation of different phenotypes by nutrient compounds ${ }^{(32,33)}$. However, the current paradigm is to study the overall dietary pattern, instead of providing a simple assessment of isolated nutrients, because of the synergistic or antagonistic effects of food items and nutrients ${ }^{(34)}$. After analysing the overall Mediterranean dietary pattern, we wanted to study the individual items that make up the MD and contribute to the modulation of the genetic effect. As we reported above, six items of the MD were significant, which agrees with the synergistic or antagonistic effects of food items in relation to metabolic diseases.

The benefits of the high consumption of vegetables in cardiovascular intermediate phenotypes have been reported in many studies ${ }^{(35)}$. In Mediterranean countries, vegetable consumption is linked to a high consumption of olive oil because it is used to dress salads. Therefore, its role in the prevention of the genetic risk for TAG concentrations could actually be associated with olive oil consumption.

Meat products and butter have a high content of SFA, and SFA have also been associated with cardiovascular risk ${ }^{(36)}$. Therefore, LL carriers could find more benefits from a low consumption of these products to decrease their TAG concentrations. 
There are some limitations in the present study, mainly derived from the fact that cross-sectional data have been analysed; therefore, causal inference cannot be made.

In conclusion, we found that the P446L SNP in the GCKR gene is significantly associated with TAG concentrations in this Mediterranean population, in agreement with previous findings. We also showed combined gene-gene associations with TAG concentrations. We found, for the first time, that the Mediterranean dietary pattern could modulate the effects of the GCKR gene variation on TAG concentrations. We also identified the main food items of this pattern that exert their beneficial effects on TAG concentrations (high consumption of olive oil, nuts and vegetables and lower consumption of red meat, butter and sweetened beverages). The present findings suggest that the adoption of the MD may decrease TAG concentrations, especially among the genetically highrisk population. However, more studies analysing dietary patterns are recommended to better establish personalised recommendations.

\section{Acknowledgements}

This study was supported by grants from the Ministerio de Ciencia e Innovación and Fono Europeo de Desarrollo Regional: CIBER CB06/03/0035, PI07-0954, CNIC-06 and AGL2010-22319-C03-03, PFU-AP2007-04663 and the Generalitat Valenciana (GVACOMP2011-151 and AP111/10). The authors' contributions are as follows: D. C. conceptualised and designed the study; M. S.-P., M. G., J. V. S., O. P., P. G.-S., J. I. G. and D. C. were involved in the acquisition of the data; M. S.P., L. Q. and D. C. analysed and interpreted the data; M. S.-P. and D. C. drafted the manuscript; M. S.-P., M. G., J. V. S., O. P., P. G.-S., J. I. G., L. Q. and D. C. critically revised the manuscript for important intellectual content. The authors declare that there are no conflicts of interest.

\section{References}

1. Grimsby J, Coffey JW, Dvorozniak MT, et al. (2000) Characterization of glucokinase regulatory protein-deficient mice. J Biol Chem 17, 7826-7831.

2. Slosberg ED, Desai UJ, Fanelli B, et al. (2001) Treatment of type 2 diabetes by adenoviral-mediated overexpression of the glucokinase regulatory protein. Diabetes 50, 1813-1820.

3. Farrelly D, Brown KS, Tieman A, et al. (1999) Mice mutant for glucokinase regulatory protein exhibit decreased liver glucokinase: a sequestration mechanism in metabolic regulation. Proc Natl Acad Sci U S A 965, 14511-14516.

4. Saxena R, Voight BF, Lyssenko V, et al. (2007) Genome-wide association analysis identifies loci for type 2 diabetes and triglyceride levels. Science 316, 1331-1336.

5. Orho-Melander M, Melander O, Guiducci C, et al. (2008) Common missense variant in the glucokinase regulatory protein gene is associated with increased plasma triglyceride and C-reactive protein but lower fasting glucose concentrations. Diabetes 57, 3112-3121.

6. Qi Q, Wu Y, Li H, et al. (2009) Association of GCKR rs780094, alone or in combination with GCK rs1799884, with type 2 diabetes and related traits in a Han Chinese population. Diabetologia 52, 834-843.
7. Sparsø T, Andersen G, Nielsen T, et al. (2008) The GCKR rs780094 polymorphism is associated with elevated fasting serum triacylglycerol, reduced fasting and OGTTrelated insulinaemia, and reduced risk of type 2 diabetes. Diabetologia 51, 70-75

8. Dupuis J, Langenberg C, Prokopenko I, et al. (2010) New genetic loci implicated in fasting glucose homeostasis and their impact on type 2 diabetes risk. Nat Genet 42, 105-116.

9. Vaxillaire M, Cavalcanti-Proença C, Dechaume A, et al. (2008) The common P446L polymorphism in GCKR inversely modulates fasting glucose and triglyceride levels and reduces type 2 diabetes risk in the DESIR prospective general French population. Diabetes 57, 2253-2257.

10. Vaxillaire M, Veslot J, Dina C, et al. (2008) Impact of common type 2 diabetes risk polymorphisms in the DESIR prospective study. Diabetes 57, 244-254.

11. März W, Nauck M, Hoffmann MM, et al. (2004) G(-30)A polymorphism in the pancreatic promoter of the glucokinase gene associated with angiographic coronary artery disease and type 2 diabetes mellitus. Circulation 15, 2844-2849.

12. Rose CS, Ek J, Urhammer SA, et al. (2005) - 30G > A polymorphism of the beta-cell-specific glucokinase promoter associates with hyperglycemia in the general population of whites. Diabetes 54, 3026-3031.

13. Yamada K, Yuan X, Ishiyama S, et al. (1997) Clinical characteristics of Japanese men with glucokinase gene beta-cell promoter variant. Diabetes Care 20, 1159-1161.

14. Estruch R, Martínez-González MA, Corella D, et al. (2006) Effects of a Mediterranean-style diet on cardiovascular risk factors: a randomized trial. Ann Intern Med 145, 1-11.

15. Nettleton JA, McKeown NM, Kanoni S, et al. (2010) Interactions of dietary whole-grain intake with fasting glucoseand insulin-related genetic loci in individuals of European descent: a meta-analysis of 14 cohort studies. Diabetes Care 33, 2684-2691.

16. Perez-Martinez P, Delgado-Lista J, Garcia-Rios A, et al. (2011) Glucokinase regulatory protein genetic variant interacts with omega-3 PUFA to influence insulin resistance and inflammation in metabolic syndrome. PLoS One; 6, e20555.

17. Elosua R, Garcia M, Aguilar A, et al. (2000) Validation of the Minnesota Leisure Time Physical Activity Questionnaire in Spanish women. Investigators of the MARATHON Group. Med Sci Sports Exerc 32, 1431-1437.

18. Fernández-Ballart JD, Piñol JL, Zazpe I, et al. (2010) Relative validity of a semi-quantitative food-frequency questionnaire in an elderly Mediterranean population of Spain. Br J Nutr 103, 1808-1816.

19. Mataix J (2003) Tabla de composición de alimentos (Food Composition Tables). Granada: University of Granada.

20. Schröder H, Fitó M, Estruch R, et al. (2011) A short screener is valid for assessing Mediterranean diet adherence among older Spanish men and women. J Nutr 141, 1140-1145.

21. Tam CH, Ma RC, So WY, et al. (2009) Interaction effect of genetic polymorphisms in glucokinase (GCK) and glucokinase regulatory protein (GCKR) on metabolic traits in healthy Chinese adults and adolescents. Diabetes $\mathbf{5 8}$, $765-769$.

22. Tam CH, Ho JS, Wang Y, et al. (2010) Common polymorphisms in MTNR1B, G6PC2 and GCK are associated with increased fasting plasma glucose and impaired beta-cell function in Chinese subjects. PLoS One 8, e11428.

23. Takeuchi F, Katsuya T, Chakrewarthy S, et al. (2010) Common variants at the GCK, GCKR, G6PC2-ABCB11 and MTNR1B loci are associated with fasting glucose in two Asian populations. Diabetologia 53, 299-308. 
24. Beer NL, Tribble ND, McCulloch LJ, et al. (2009) The P446L variant in GCKR associated with fasting plasma glucose and triglyceride levels exerts its effect through increased glucokinase activity in liver. Hum Mol Genet 18, 4081-4088.

25. Webster RJ, Warrington NM, Weedon MN, et al. (2009) The association of common genetic variants in the APOA5, LPL and GCK genes with longitudinal changes in metabolic and cardiovascular traits. Diabetologia 52, 106-114.

26. Hu C, Zhang R, Wang C, et al. (2010) Effects of GCK, GCKR, G6PC2 and MTNR1B variants on glucose metabolism and insulin secretion. PLoS One 5, e11761.

27. Perez-Martinez P, Corella D, Shen J, et al. (2009) Association between glucokinase regulatory protein (GCKR) and apolipoprotein A5 (APOA5) gene polymorphisms and triacylglycerol concentrations in fasting, postprandial, and fenofibrate-treated states. Am J Clin Nutr 89, 391-399.

28. Shen H, Pollin TI, Damcott CM, et al. (2009) Glucokinase regulatory protein gene polymorphism affects postprandial lipemic response in a dietary intervention study. Hum Genet 126, 567-574.

29. Estruch R (2010) Anti-inflammatory effects of the Mediterranean diet: the experience of the PREDIMED study. Proc Nutr Soc 69, 333-340.

30. Sotos-Prieto M, Zulet MA \& Corella D (2010) Scientific evidence of the Mediterranean diet effects in determining intermediate and final cardiovascular disease phenotypes. Med Clin (Barc) 134, 22-29.

31. Fenech M, El-Sohemy A, Cahill L, et al. (2011) Nutrigenetics and nutrigenomics: viewpoints on the current status and applications in nutrition research and practice. $J$ Nutrigenet Nutrigenomics 4, 69-89.

32. Corella D, Tai ES, Sorlí JV, et al. (2010) Association between the APOA2 promoter polymorphism and body weight in Mediterranean and Asian populations: replication of a gene-saturated fat interaction. Int $J$ Obes (Lond) 35 , 666-675.

33. Sotos-Prieto M, Guillén M, Guillem-Sáiz P, et al. (2010) The rs1466113 polymorphism in the somatostatin receptor 2 gene is associated with obesity and food intake in a Mediterranean population. Ann Nutr Metab 57, 124-123.

34. Hu FB (2002) Dietary pattern analysis: a new direction in nutritional epidemiology. Curr Opin Lipidol 13, 3-9.

35. Bendinelli B, Masala G, Saieva C, et al. (2011) Fruit, vegetables, and olive oil and risk of coronary heart disease in Italian women: the EPICOR Study. Am J Clin Nutr 93, 275-283.

36. Polychronopoulos E, Pounis G, Bountziouka V, et al. (2010) Dietary meat fats and burden of cardiovascular disease risk factors, in the elderly: a report from the MEDIS study. Lipids Health Dis 9, 30. 